Print ISSN: $2735-4121$

Online ISSN : $2735-413 \mathrm{X}$

\title{
Effect of Eye Care Learning Package for Mechanically Ventilated Patients on Critical Care Nurses' Performance
}

\author{
WardaElmoghazyElkasby ،*( $)$ WafaaWahdanAbd El-Aziz‘ $\left({ }^{\Upsilon}\right)$ WafaaGameel Mohamed Ali $\left({ }^{\Gamma}\right)$ \\ 1 Head Nurse in Mansoura University Hospital, Egypt *Corresponding author \\ 2Lecturer of Critical Care and Emergency Nursing Department, Faculty of Nursing, Mansoura \\ University, Egypt \\ 3Prof of Medical-Surgical Nursing, Faculty of Nursing, Mansoura University, Egypt \\ *Corresponding author: WardaElmoghazyElkasby (wardaelmoghazy@yahoo.com).
}

\begin{abstract}
Background: Eyecare is an important aspect of nursing care for critical cases, but is usually ignored due to continuous stress on life-threatening conditions in those patients. Aim: To examine the effect of eye care learning package for mechanically ventilated patients on critical care nurses' performance. Method: Quasi-experimental research design was utilized to conduct this study. Sample: A convenience sample of 50 nurses working at the Main Mansoura University Hospitals was included in this study. Tools: Data were collected using two tools. Tool I: Eye Care Knowledge Questionnaire. This tool included two parts; part 1 covered the socio-demographic characteristics of the studied nurses, and part 2 involved an eye care knowledge questionnaire. The second tool was the eye care observational checklist. Results: after learning package implementation; there was a highly statistically significant improvement in critical care nurses' performance regarding eye care for mechanically ventilated patients. Conclusion: the findings of the current study revealed statistically significant improvements in critical care nurses' performance postimplementation of the learning package compared to pre-education. Recommendations: Based on the current study findings, continuous training programs for critical care nurses' regarding eye care for mechanically ventilated patients are recommended to improve the patients' outcomes. Additionally, eye assessment should be part of the daily routine physical assessment and care of mechanically ventilated patients.

Keywords Critically Ill Patients, Eye Care, Nurses' Knowledge, Mechanically Ventilated Patients; Performance.
\end{abstract}




\section{Introduction}

Intensive care patients receiving mechanical ventilation (MV), sedation, and/or paralytic medications have an increased chance of acquiring a hospitalacquired ocular infection secondary to their impaired natural eye defense mechanisms (Grixti, Sadri, Edgar \& Datta, 2012). The eyes normally flush microorganisms out and aids in distributing tears to lubricate the ocular surface. Unfortunately, patients on MV are at high risk of ocular infection. In addition to, prolonged eye-opening which exposes the patient to permanent ocular damage and infection (Azfar, Khan \& Alzeer, 2013; Dawson, 2005; Mela et al., 2010).

Eyecare is an important concern of nursing care for critical cases that are at high risk of eye injury due to their sickness and management can compromise ocular protective mechanisms. Critical care nurses (CCNs') and physicians in the intensive care units (ICUs) often stress on conditions which are life-threatening emergency most of the time that cause low interest of the less emergency conditions such as problems affecting the eyes that may be a serious situation (Rosenberg \& Eisen, 2008). Australian Nursing Federation (2008) in the best performance information sheet confirms that care of the patient eyes is part of the care delivered to all patients in acute care with impaired or compromised protective mechanisms. The loss of conscious patients, given sedation or paralysis considered high-risk personnel who need eye care to keep the integrity of eyes.

Also, patients in the ICUs are at high risk for eye injury secondary to the disturbance of the defense mechanism of the eye and the drawback effect of the $\mathrm{MV}$ and use of sedative drugs which lower lacrimation release and increase evaporation secondary to prolonged eyeopening (Azfar et al., 2013; Fashafsheh, Morsy, Ismaeel \& Alkaias, 2013).

Furthermore, many causes predispose patients to impaired ocular defense mechanism such as a disturbance conscious level, affecting on the reflex and, metabolic process, immune suppression, artificial ventilation, drugs such as sedation, muscle relaxation drugs and paralysis, open suction method, and systemic disease (Marshall, Elliott, Rolls, Schacht \& Boyle, 2008; Yim, 2009).

One of the major challenges that may face CCNs is to be competent in both using a wide range of technological devices and meeting patient needs. Therefore, care of the eye is a vital concern of the nursing care of patients with critical situations, particularly for those patients whose ocular protective mechanisms may be compromised (Azfar, et al., 2013).

Moreover, the role of CCNs' in the ICUs should be focused on eye assessment to evaluate any sign of eye infection such as dry eye, swelling, and conjunctiva redness; perform eye cleaning with saline-soaked gauze to clean secretion; debris or dried ocular medications every two hours and use of eye cover. Also, nurses should use ways of restricting the aerostation of secretions like the tracheal suction technique and avoid suction catheters touch the patient's eyes (Werli-Alvarenga, Ercole, Herdman \& Chianca, 2012).

Critical care nurses play an
important role in delivering comprehensive care for patients with a critical illness. They are well prepared to apply continuous follow-up and 
advanced care for different critical illness situations (Bell, 2015). The nurse-patient ratio in the ICU is mostly 1 to 2 . Moreover, CCNs' are in a position to deliver continuous assessment and early detect any abnormality in a patient with critical cases that requires proper management (Hu et al., 2017). They should also have critical thinking and problem-solving skills, and evidencebased nursing practice and research to provide high-quality patient care (Critical Care Services Ontario, 2012).

Eyecare is an important concern of a daily nursing care plan. It includes regular eye assessment and prevention of iatrogenic ophthalmological complications by cleaning the eye with normal saline or sterile water, close the eyelid using ocular lubricant, or creation a moisture chamber using polyethylene wrap. Therefore, the ICU nurses should have great attention to the patients' care with critical health conditions which necessitate individual and advanced interventions to satisfy patient's needs (Milutinovic, Cvijanovic, Ciric, Jovanovic \& Andrijevic, 2017). So, the current research was conducted to investigate the effect of eye care learning packages for mechanically ventilated patients on the CCNs' performance

Significance of the study

Through my clinical observation, it was found that nurses' knowledge regarding eye care for MV patients has not been assessed, and little is identified about the relationship between CCNs' knowledge and their effect on practices to prevent ocular complications in critically ill patients. Also, lack of evidence-based practice and insufficient training has resulted in uncertainty and knowledge deficit regarding eye care. This urged the importance to enhance CCNs' knowledge and practices of eye care for MV patients. Educating and training nurses in this area will enhance their skills and knowledge, and consequently improve patients' outcomes (Fashafsheh et al., 2013; Milutinovic et al., 2017). Therefore, this research was conducted to investigate the effect of eye care learning packages for MV patients on CCNs' performance. Eventually, this research might generate attention and motivation for further research studies in this area.

\section{Aim of the Study:}

The study aimed to examine the effect of eye care learning package for mechanically ventilated patients on critical care nurses' performance.

\section{Research hypotheses}

To achieve the study, aim the following research hypotheses were formulated

- The mean scores of CCNs' knowledge who are exposed to the learning package for eye care will be high score than before.

- The mean scores of CCNs' practice who are exposed to the learning package for eye care will be high score than before.

\section{Subject and Methods}

Research design: Quasiexperimental pre-post one group research design was used in this study. Setting: This study was carried out at two ICUs at the Main Mansoura University Hospitals (MUHs), Egypt. Surgical ICU and Anesthesia ICU, Surgical ICU of the Critical Care and Convalescence Hospital at Mansoura University includes two rooms with nine beds. The unit is well equipped with advanced technology and manpower required for patients' management such as MV, bedside monitors, infusion pumps, suction machines, and a portable x-ray machine. According to the hospital's records, the unit receives about 500 
patients annually from Mansoura and its surroundings. This unit provides care for patients after major surgeries such as prostate surgery, cholecystectomy, a partial colectomy, or amputation. It also receives patients with postoperative complications such as sepsis, respiratory failure, and severe bleeding. The nursepatient ratio in this ICU is nearly 1:2. Anesthesia ICU at Mansoura University hospitals include eight-bed. It is well equipped with advanced technology and manpower required for patients' management such as MV, bedside monitors, infusion pumps, suction machines, arterial blood gases (ABG) machine, and a portable $\mathrm{x}$-ray machine. It provides care for patients after major surgeries such as hysterectomy, placenta previa, accidental hemorrhage, intestinal obstruction, and mesenteric vascular occlusion (MVO). It also receives patients with postoperative complications such as respiratory failure, and severe bleeding. The nurse-patient ratio in this ICU is nearly $1: 2$.

Study sample: A convenience sample of 50 nurses working in the selected ICUs with various education levels, who had at least one year of work experience and were involved in delivering direct care to critically ill patients, was selected to achieve the aim of the present research.

Tools of the study: Two tools were developed by the researcher after vigorous review of the related literature (Alghamdi, Ghaleb \& Abdelaa, 2018; Fashafsheh et al., 2013; Milutinovic et al., 2017) to assess CCNs' knowledge and performance concerning eye care of the patient on the mechanical ventilator. Two tools were used to gather data pertinent to the study as follows: Tool I: Eye Care Knowledge Questionnaire; it consisted of two parts as follows: Part
A: Nurses' socio-demographic characteristics; such as age, gender, marital status, level of education, occupation, and years of experience in the ICU.

Part II: Eye Care Knowledge Questionnaire: It composed of 26 items of multiple-choice questions which divided into four main knowledge domains; this tool including 11 items for nurses' knowledge about anatomy and physiology of the eye, mechanical ventilation complications 5 items, medications effect on the eye health status of the patients 3 items, and 7 items regarding eye infection.

Scoring system: Nurses' knowledge items were evaluated as the following, the correct answer was given one score and the incorrect answer was given zero scores. The total scoring of CCNs' knowledge was ranged from 1 to 26. They were calculated and classified as; less than $50 \%$ were considered a poor level of knowledge, $50 \%$ to $65 \%$ were considered a fair level of knowledge, and more than $65 \%$ were considered a good level of knowledge.

Tool II: Eye Care Observational Checklist: This tool was used to assess CCNs' practice in ICU during providing eye care for mechanically ventilated patients. It is composed of 28 items and divided into three main domains; 7 steps for eye assessment, 12 steps for infection control activities, and eye care management action 9 steps.

Scoring system: Nurses' practice steps were rated on 3 points Likert scale; 2 for each completely correct step, 1 for partially correct step, and zero for incorrect step. The total score of practice was ranged from 0 to 56 . The higher scores refer to higher performance levels. They were categorized as: scores equal 
or more than $60 \%$ were considered as adequate performance level and scores lower than $60 \%$ refer to inadequate performance level.

\section{Methods}

All tools of this study were developed by the researchers after a strong review of the related literature and utilized to gather the needed information. The tool I of data collection was translated into the Arabic language and back translation was done because the studied nurses had different levels of education.

All tools were tested for content validity by five panels of juries' expertise in the field of critical care nursing and medical biostatistics and modifications were applied.

All tools of the study were tested for reliability using Cronbach alpha. The reliability of tool I and tool II was 0.819 and 0.927 respectively which represents a highly reliable tool.

A pilot study: A pilot study was conducted before beginning collection of data on $10 \%$ of studied nurses to check it for the clarity, feasibility, and applicability of the tools as well as to detect the time needed to gather the needed information.

\section{Ethical consideration:}

Official permission was taken before starting the current research from Mansoura University Hospital administrator. The research procedure was reviewed and accepted by the Ethical Committee of the Faculty of Nursing at Al Mansoura University. Written approval was achieved from studied nurses after the researchers clarified the aim of the current study. Privacy and data confidentiality were maintained by all studied nurses. The studied nurses had the right to withdraw from the present research at any time of the research process.

\section{Data collection:}

The studied subject who willing to participate in this research have been interviewed and observed by the researchers. The data collection duration was extended from the period of the starting of January to the end of August (2019).

The current study was carried out in four phases including the assessment, planning, implementation, and evaluation phases.

Assessment phase: It was performed for all studied nurses before learning package implementing as a pretest to assessed CCNs' knowledge about eye care, using the tool I and lasted for 30-35 minutes, throughout three shifts, for about two months from the first of January 2019 to the end of February 2019. Also, direct observation was done by the investigator to assess nurses' performance regarding eye care using tool II. Privacy and confidentiality of the gathered data were maintained and assured.

Planning phase: During this phase, a learning package was formulated focused on the assessment phase and an extensive review of the relevant literature (Alghamdi et al., 2018; Fashafsheh et al., 2013; Khalil et al., 2019). Goals and expected outcome criteria were being formulated based on priorities during implementing learning packages the following: improving nurses' total knowledge and practice mean scores regarding eye care for $\mathrm{MV}$ patients.

Implementation phase: During this phase, the learning package was applied to all studied nurses during sessions. These sessions were done in the educational classroom beside ICU at 
MUHs. The nurses were distributed into subgroups. Every subgroup ranged between 8 and 10 nurses.

The six sessions were divided to three sessions conducted on three consecutive days for the theoretical part. Others three sessions were conducted for the following three consecutive days for the clinical section. Every session consumed about 40- 60 minutes. The session time was between morning and afternoon shift or through morning shift after applying the routine care to the patients with critical conditions.

.For the theoretical part; three sessions were included; eye anatomy and physiology, MV complications, and sedatives \& neuro blocker effects on the eye of ventilated patients.

For the clinical part; three clinical sessions were utilized following theoretical sessions for 3 consecutive days. Session one consisted of infection control guidelines related to eye care, session two included assessment and examination of the eye, session three involving documentation and reporting findings.

The practical sessions were conducted in the ICU, demonstration and re-demonstration methods were applied for each nurse. The teaching media used in this research were videos, moderate fidelity simulation, and problem-solving situations.
Evaluation phase: the studied nurses were evaluated using tools I and II. Each nurse was evaluated separately in the ICU two times. The first evaluation (pretest evaluation) was conducted before implementing the learning package to evaluate the nurses' knowledge and performance regarding eye care for mechanically ventilated patients. Then the total knowledge and practice mean scores were calculated. The second evaluation (posttest evaluation) was done after implementing the learning package to evaluate the nurses' knowledge and practice regarding eye care. Then the total knowledge and practice mean scores were calculated separately.

Statistical Analysis:

All statistical tests were conducted using SPSS for windows version 20.0 (SPSS, Chicago, IL). Continuous data were presented in mean score \pm standard deviation (SD). Categorical data were expressed in frequency and percentage. The comparisons were determined using the independent t-test for two variables. A Chi-square test was used for relationships between variables. Statistical significance was set at $\mathrm{p}<0.05$. Results

Table 1: Illustrated that the majority of studied nurses $(78 \%)$ were at age 25-29 years. More than half of the studied samples $(58 \%)$ were female and more than half of the nurses $(66 \%)$ were married. Also more than half of nurses $(52 \%)$ had achieved a high level of education. 
Effect of Eye Care Learning Package etc...

Table (1): Percentage distribution of studied nurses regarding personal characteristics

\begin{tabular}{|c|c|c|}
\hline Nurses' characteristics & $\begin{array}{c}\text { No. } \\
(\mathrm{n}=50)\end{array}$ & $\%$ \\
\hline \multicolumn{3}{|l|}{ Age } \\
\hline years r925 - & rq & VA. \\
\hline 30 or more years & 11 & Tr. \\
\hline \multicolumn{3}{|c|}{$r .{ }^{\top} \pm r V .{ }^{q}$ Mean \pm SD } \\
\hline \multicolumn{3}{|l|}{ Gender } \\
\hline Male & YI & $\varepsilon r_{.} \cdot$ \\
\hline Female & rq & $0 \wedge$. \\
\hline \multicolumn{3}{|l|}{ Marital status } \\
\hline Married & r & 74. \\
\hline Single & 10 & $r \cdot . \cdot$ \\
\hline Divorce & T & ¿. \\
\hline \multicolumn{3}{|l|}{ Educational level } \\
\hline Diploma & 11 & Tr. \\
\hline Technical & 14 & YY. \\
\hline Bachelor & YT & or.. \\
\hline \multicolumn{3}{|l|}{ Experience } \\
\hline years ${ }^{\circ}>$ & KY & $\varepsilon \varepsilon_{.}$ \\
\hline years $1 \cdot 5-$ & YY & $\varepsilon \varepsilon$. \\
\hline years $1 \cdot<$ & 7 & Tr. \\
\hline \multicolumn{3}{|c|}{$r . V_{ \pm} \cdot \wedge$ Mean \pm SD } \\
\hline
\end{tabular}

Table 2: Show the difference between total knowledge score regarding the eye care among studied nurses prepost learning package implementation. It showed that there are a significant statistical difference between CCNs' knowledge regarding (anatomy and physiology, mechanical ventilation Table (2): The difference between total knowledge score regarding the eye care among studied nurses pre post-learning package implementation

\begin{tabular}{|l|c|c|c|}
\hline \multicolumn{1}{|c|}{ Knowledge domains } & $\begin{array}{c}\text { Pre learning package } \\
\text { implementation }\end{array}$ & $\begin{array}{c}\text { Post learning package } \\
\text { implementation }\end{array}$ & $\begin{array}{c}\text { Paired T- } \\
\text { test } \\
\text { t-test } \\
\text { Pean } \pm \text { SD }\end{array}$ \\
\cline { 2 - 4 } & $\mathbf{4 . 1} \pm \mathbf{1 . 7}$ & Mean \pm SD & $\begin{array}{c}\mathbf{1 2 . 1 3 4} \\
<\mathbf{0 . 0 0 1} * *\end{array}$ \\
\hline $\begin{array}{l}\text { Anatomy and physiology of } \\
\text { the eye }\end{array}$ & $\mathbf{3 . 0} \pm \mathbf{1 . 3}$ & $9.2 \pm 2.1$ & $\mathbf{3 . 8 4 6}$ \\
\hline $\begin{array}{l}\text { Mechanical ventilation } \\
\text { complications }\end{array}$ & $\mathbf{1 . 4} \pm \mathbf{0 . 9}$ & $3.8 \pm 0.8$ & $\mathbf{0 . 0 0 1} * *$ \\
\hline $\begin{array}{l}\text { Effect of Nueromuscular } \\
\text { medications }\end{array}$ & $\mathbf{3 . 6} \pm \mathbf{1 . 6}$ & $\mathbf{6 . 8 9 3} \pm \mathbf{1 . 2}$ & $<\mathbf{0 . 0 0 1 * *}$ \\
\hline Eye infection & & & $\mathbf{8 . 8 4 9}$ \\
\hline
\end{tabular}

$* *$ Highly statistical significant at $p \leq 0.0 \mathrm{~s} 01$ complications, effect of neuromuscular medication, and eye infection (p. value $<\mathbf{0 . 0 0 1 )}$ respectively. This means acceptance of research hyposis which stated that the mean scores of the CCNs' knowledge of who are exposed to the learning package for eye care for $\mathrm{MV}$ patients had high score than before . 
WardaElmoghazyElkasby et. al.

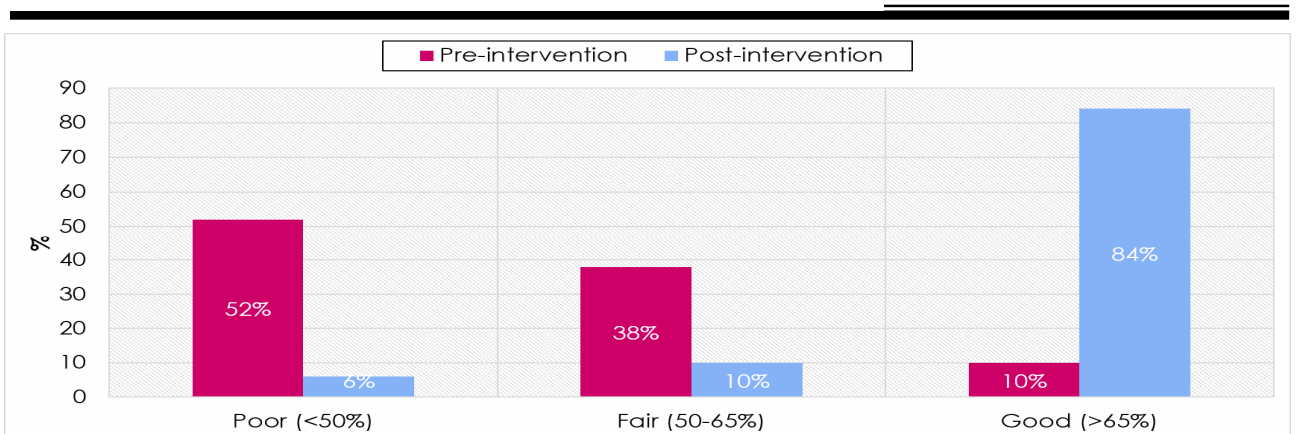

Figure $1 \mathrm{CCNs}$ total knowledge regarding eye care for MV patients before and after learning package implementation

Figure (1): Illustrates that the CCNs' significant improvement in $\mathrm{CCNs}^{\prime}$ total total knowledge regarding eye care for knowledge regarding eye care for MV MV patients before and after learning patients after learning package package. As shown there was a implementation (from $10 \%$ to $84 \%$ ).

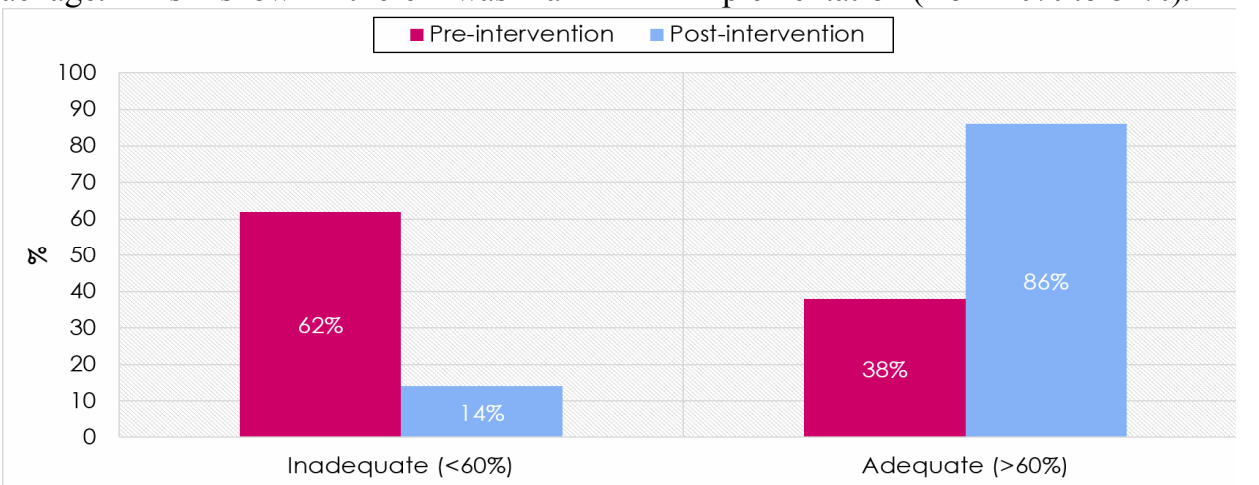

Figure 2. CCNs' total performance before and after learning package implementation.

Figure (2): Illustrates that the CCNs' total performance regarding eye care for MV patients before and after learning package. As shown there was highly statistical significant improvement in CCNs' total performance regarding eye care for MV patients after learning package implementation $(\mathrm{P}-<0.001$. (

Table (3): Show the relationship between the total knowledge score and

Table (3): The relationship between the total knowledge score and the total practice score pre learning package implementation

\begin{tabular}{|l|l|l|l|l|l|l|l|l|}
\hline Practice & \multicolumn{9}{|c|}{ Knowledge } & \multicolumn{2}{c|}{} \\
\hline & \multicolumn{2}{|c|}{ Low } & \multicolumn{2}{c|}{ Moderate } & \multicolumn{2}{c|}{ High } & \multicolumn{2}{c|}{ Chi-square test } \\
\cline { 2 - 10 } & $\mathrm{N}$ & $\%$ & $\mathrm{~N}$ & $\%$ & $\mathrm{~N}$ & $\%$ & $\mathrm{X}^{2}$ & $\mathrm{P}$ \\
\hline Inadequate & 24 & 92.3 & 7 & 36.8 & 0 & 0.0 & & \\
\hline Adequate & 2 & 7.7 & 12 & 63.2 & 5 & 100.0 & 23.399 & $<\mathbf{0 . 0 0 1}$ \\
\hline
\end{tabular}


Table (4): Shows the relationship between personal characteristics of the studied nurses and level of total knowledge score regarding eye care for mechanical ventilated patients pre learning package implementation. This finding illustrated that there is positive statistically significant relationship between total knowledge score and age, educational level and years of experience of the study sample (P value $\mathbf{0 . 0 0 3 , 0 . 0 2}$ and 0.09 respectively). This means that the participant nurses who older, highly educated and has more years of experience tend to be more knowledgeable than others

Table (4): The relationship between personal characteristics of the studied nurses and level of total knowledge score pre learning package implementation regarding eye care for mechanically ventilated patients.

\begin{tabular}{|c|c|c|c|c|c|c|c|}
\hline \multirow[t]{3}{*}{ Nurses' characteristic } & \multicolumn{6}{|c|}{ Pre total knowledge score } & \multirow{3}{*}{$\underset{P}{X^{2}}$} \\
\hline & \multicolumn{2}{|c|}{ Low } & \multicolumn{2}{|c|}{ Moderate } & \multicolumn{2}{|c|}{ High } & \\
\hline & $\mathrm{N}$ & $\%$ & $\mathrm{~N}$ & $\%$ & $\mathrm{~N}$ & $\%$ & \\
\hline \multicolumn{8}{|l|}{ Age } \\
\hline $25-29$ yrs & 25 & 96.2 & 12 & 63.2 & 2 & 40.0 & \multirow{2}{*}{$\begin{array}{l}11.64 \\
0.003 *\end{array}$} \\
\hline 30 or more yrs & 1 & 3.8 & 7 & 36.8 & 3 & 60.0 & \\
\hline \multicolumn{8}{|l|}{ Gender } \\
\hline Male & 12 & 46.2 & 7 & 36.8 & 2 & 40.0 & \multirow{2}{*}{$\begin{array}{l}0.400 \\
0.81\end{array}$} \\
\hline Female & 14 & 52.8 & 12 & 63.2 & 3 & 60.0 & \\
\hline \multicolumn{8}{|l|}{ Marital status } \\
\hline Married & 16 & 61.5 & 15 & 78.9 & 2 & 40.0 & \multirow{3}{*}{$\begin{array}{l}4.31 \\
0.36\end{array}$} \\
\hline Single & 9 & 34.6 & 3 & 15.8 & 3 & 60.0 & \\
\hline Divorce & 1 & 3.8 & 1 & 5.3 & 0 & 0.0 & \\
\hline \multicolumn{8}{|l|}{ Educational level } \\
\hline Diploma & 5 & 19.2 & 5 & 26.3 & 1 & 20.0 & \multirow{3}{*}{$\begin{array}{l}12.01 \\
0.02 *\end{array}$} \\
\hline Technical & 12 & 46.2 & 1 & 5.3 & 0 & 0.0 & \\
\hline Bachelor & 9 & 34.6 & 13 & 68.4 & 4 & 80.0 & \\
\hline \multicolumn{8}{|l|}{ Experience } \\
\hline$<5$ years & 16 & 61.5 & 5 & 26.3 & 1 & 20.0 & \multirow{3}{*}{$\begin{array}{l}7.96 \\
0.09 *\end{array}$} \\
\hline $5-10$ years & 9 & 34.6 & 10 & 52.6 & 3 & 60.0 & \\
\hline$>10$ years & 1 & 3.8 & 4 & 21.1 & 1 & 20.0 & \\
\hline
\end{tabular}

Table (5): Shows the relationship between personal characteristics of the studied nurses and level of total practice score regarding eye care for mechanical ventilated patients pre learning package implementation. This finding illustrated that there were strong positive statistical significant relationships between total practice score and nurses' age, educational level and years of experience of the study sample ( $P$ value $<\mathbf{0 . 0 0 1}$, 0.02 and 0.002 respectively). This means that the participant nurses who older, highly educated and had more years of experience tend to be more skillful than others. 
Table (5): The relationship between personal characteristics of the studied nurses and level of total practice score pre learning package implementation regarding eye care for mechanical ventilated patients

\begin{tabular}{|c|c|c|c|c|c|}
\hline \multirow[t]{3}{*}{ Nurses' characteristics } & \multicolumn{4}{|c|}{ Pre total practice score } & \multirow{4}{*}{$\underset{P}{X^{2}}$} \\
\hline & \multicolumn{2}{|c|}{ Inadequate } & \multicolumn{2}{|c|}{ Adequate } & \\
\hline & $\mathrm{N}$ & $\%$ & $\mathrm{~N}$ & $\%$ & \\
\hline \multicolumn{5}{|l|}{ Age } & \\
\hline $25-29$ yrs & 30 & 96.8 & 9 & 47.4 & \multirow{2}{*}{$\begin{array}{c}16.75 \\
* * 0.001>\end{array}$} \\
\hline 30 or more yrs & 1 & 3.2 & 10 & 52.6 & \\
\hline \multicolumn{6}{|l|}{ Gender } \\
\hline Male & 13 & 41.9 & 8 & 42.1 & \multirow{2}{*}{$\begin{array}{l}0.001 \\
0.991 \\
\end{array}$} \\
\hline Female & 18 & 58.1 & 11 & 57.9 & \\
\hline \multicolumn{6}{|l|}{ Marital status } \\
\hline Married & 19 & 61.3 & 14 & 73.7 & \multirow{3}{*}{$\begin{array}{l}1.21 \\
0.54\end{array}$} \\
\hline Single & 11 & 35.5 & 4 & 21.1 & \\
\hline Divorce & 1 & 3.2 & 1 & 5.3 & \\
\hline \multicolumn{6}{|l|}{ Educational level } \\
\hline Diploma & 5 & 16.1 & 6 & 31.6 & \multirow{3}{*}{$\begin{array}{c}7.08 \\
* 0.02\end{array}$} \\
\hline Technical & 12 & 38.7 & 1 & 5.3 & \\
\hline Bachelor & 14 & 45.2 & 12 & 63.2 & \\
\hline \multicolumn{6}{|l|}{ Experience } \\
\hline years 5> & 19 & 61.3 & 3 & 15.8 & \multirow{3}{*}{$\begin{array}{c}12.12 \\
* * 0.002\end{array}$} \\
\hline years $10-5$ & 11 & 35.5 & 11 & 57.9 & \\
\hline years $10<$ & 1 & 3.2 & 5 & 26.3 & \\
\hline
\end{tabular}

*Correlation is significant at the 0.05

\section{level **Highly statistical}

significant at $p \leq 0.001$

\section{Discussion}

Critical care nurses play an important role in a health caregiver team, with the cooperation of the doctors to save the patient's life. The ICU nursing staff usually early detect patients' problems, that require rapid concern, since it is a life-threatening condition that may lead to poor of attention to other serious conditions like the care of eyes (Alghamdi et al., 2018).

The study has been included a sample of fifty nurses. Regarding studied nurses' qualifications, more than half of participant nurses were holding bachelor's degrees. This might be because of elevated percentage of employment of bachelor degree of nurses in selected ICUs and MUHs hospital administration prefer mostly CCNs' have a bachelor degree. This consistent with Alghamdi et al. (2018); Gluer, Eşer \& Fashafsheh (2017), they found that the majority of the studied nurses in ICUs had bachelor's degrees. On the other hand, this result was contradicted with Botros, Mohamed \& Ahamed (2019) their study conducted to assess nursing practice regarding safty measures on MV patients and found that the whole nursing staff in the field of study was diploma nurses and technical nurses.

In relation to age of study participants, more than two- third of participant nursing staff aged from 25 to 29 years. This can explained by the presence of newly graduate nurses in selected ICUs. This was in agreement with (Aslam, Afzal, Kousar, Waqas \& Gilani (2017).) they found that most of nurses were aged 18 - 29 years. 
Regarding CCNs' years of work experience in ICUs, the present study revealed that nearly half of studied nurses had 5 - 10 years of experience. This was consistent with finding of Zanaty, Morsy, Elshamy\& Ali (2016) who researched knowledge and practices concerning sepsis in the same study setting.

Regarding participant nurses knowledge regarding ocular anatomical and physiological features before learning package implementation. It was found that most of nurses had a reduced level of knowledge. From my point of view, this could be due to insufficient basic information about anatomy and physiology of the eye. This came in agreement with Taha \& Abd Elaziz (2015) which revealed that most of nursing staff showed low knowledge level regarding ocular anatomical and physiological features. While knowledge of the majority of studied nurses regarding anatomy and physiology of the eye has been improved after learning package implementation and this finding was consistent with Mohamed, Abdl Elmoniem, El-Mowafi, \& Shebl (2019). who showed that the level of knowledge was improved after implementation of the training program than before.

The present study found that majority of nurses had high knowledge level about the effect of sedatives and neuromuscular blocking drugs on the eye of MV patients after learning package implementation in comparing with their level of knowledge before. This finding consistent with (Guler et al., 2017; Milutinović et al., 2017) they found that three quarters of participant nurses acknowledged about the effect of sedatives and neuromuscular blocking drugs on physiological eye protection mechanism in MV patients.
The current study showed that good improvement in the knowledge about MV eye complications after learning package implementation. This is consistent with other previous study conducted by Fashfishi et al. (2013) who investigated the effect of a designed eye care protocol upon nurses' knowledge and practices about ocular health of comatose patient on MV and showed that good improvement in the knowledge about MV eye complications after protocol application .

The current work demonstrated that most of nurses had inadequate practice level regarding eye assessment among patients in ICUs pre learning package implementation. This is consistent with other previous study conducted by Milutinović et al. ( 2017) who found that CCN's do not have sufficient knowledge and experience in performing eye assessments. This may be caused by lack of training programs, handouts or guidelines. After learning package implementation and providing different sessions about eye assessment and availability of hand out, all of these leading to improvement in the CCN's practice regarding eye assessment.

The current study revealed that all participant nurses' didn't assess the patient for pupil reflex and eye movement by using pen light, because nurses consider this not one of their tasks and emphasizing this type of assessment is physcians roles. This finding is supported by other study conducted by Khalil, Abd Elhameed, Abdel-Kader\& Atia (2019) which illustrated that the majority of nurses were incompetent in using pen light assessment. After training and availability of hand out nearly half of studied nurses had improvement in their practice regarding to pen light 
assessment and this is consistent with Khalil et al., 2019.

Regarding infection control measures it was found that nearly half of studied nurses washed their hands incorrectly due to absence of adequate awareness about the importance of hand washing and infection control measures. This finding was also consistent with other study done by Khalil et al. (2019) who found that the majority of the studied nurses had inadequate practice level regarding hand washing. Regarding culture swap, the present study clarify there was improvement occur in performane taken during culture swap due to training program provided to CCN's, and this is consistent with Fashfishi et al. (2013) who found that the most common precaution taken by the Palestinian nurses were taking swab for culture in case of any sign of infection.

The current work showed that more than half of studied nurses had inadequate practice level as regards eye cleaning with saline pre learning package implementation. From my point of view, this could be due to insufficient basic information about eye cleaning. This was consistent with Milutinović et al. (2017) who reported that one third of studied nurses do not apply eye washing with saline solution, or perform it very rarely. While the present work revealed that the majority of studied nursing staff were competent in performing eye cleaning with saline, application of topical antibiotic or lubricant after learning package implementation. This was consistent with Vyas, Mahobia\& Bawankure (2018) studied which investigated knowledge and practice patterns of intensive care unit nurses towards eye care.
Our results showed that high percentage of studied nurses had adequate practice level in eye dropped, ophthalmic ointment, eye lid closed and the eye closed with gauze and tape horizontally post educational training implementation. This were consistent with (Ahmadinejad et al., 2020; Alansari, Hijazi\& Maghrabi, 2015) they reported ophthalmic ointment, eye closed and eye lid taping was used and examined and became routine eye care method in ICU setting.

The relationship of CCNs' knowledge and practice regarding eye care for MV patients, our finding revealed strong positive relationship between total knowledge score and total practice score pre learning package implementation. This was disagree with Khalil et al. (2019) who reported no significant correlation between total knowledge score of the studied nurses about eye care and their performance.

The current study revealed that their was a positive statistical significant relationships between CCNs' knowledge, and practices with the age and this is disagree with Fashafsheh et al., 2013.

The current study revealed that their was a positive statistical significant relationships between CCNs' knowledge, and practices with years of experience and this is consistant with Ebadi, Moayed, Mirsadeghi \& Saeid (2021) their study conducted to evaluate intensive care nurses' clinical competence in eye care and found that a significant positive correlation between nurses' work experience and their eye care knowledge and attitude.

\section{Conclusion}

The findings of the current study revealed that there were statistically significant improvements for CCNs' post-training program. This result due to 
Effect of Eye Care Learning Package etc...

a continuous services training program that improves their performance towards caring for MV patients.

\section{Recommendations}

Based on the current study findings, it is recommended that continuous training programs for CCNs' regarding eye care for mechanically ventilated patients are needed to improve the patients' outcomes. Also, assessment of the patient eyes should be part of routine and daily physical assessment and be conducted on admission and then repeated routinely at the start of the new nursing shift to be part of a complete patient care plan.

\section{References}

Ahmadinejad, M., Karbasi, E., Jahani, Y., Ahmadipour, M., Soltaninejad, M., \& Karzar, Z. (2020). Efficacy of simple eye ointment, polyethylene cover, and eyelid taping in prevention of ocular surface disorders in critically ill patients: a randomized clinical trial. Critical Care Research and Practice, pp.1-7. https://doi.org/10.1155/2020/6267 432.

Alansari, M., Hijazi, M., \& Maghrabi, K. (2015). Making a Difference in Eye Care of the Critically Ill Patients. Journal of Intensive Care Medicine, 30(6), pp. 311-31.7

Alghamdi, M., Ghaleb, M., \& Abdelaa, S. (2018). Assessment of intensive care nurse knowledge and perception of eye care practice for unconscious and mechanically ventilated patients in intensive care units in Saudi Arabia. Journal of Nursing and Health Science, 4 (1), 15-22. Retrieved from: https://www.researchgate.net.
Anf.org.au.

2021. Australian Nursing Federation. [online] Available at:

$<$ http://432anf.org.au/documents/s ubmissions/Sub National PHC St rategy.pdf $>0[$ Accessed 3 February 2021].

Aslam, S., Afzal, M., Kousar, R., Waqas, A., \& Gilani, S. (2017). The Assessment of Nurses' Knowledge and Practices about Fluid and Electrolytes Monitoring and Administration among Cardiac Surgery Patients: A Case of Punjab Institute of Cardiology. International Journal of Applied Sciences and Biotechnology, 5(2), pp.208-215.

DOI: 10.3126/ijasbt.v5i2.17626.

Azfar, M., Khan, M., \& Alzeer, A. (2013). Protocolized eye care prevents corneal complications in ventilated patients in a medical intensive care unit. Saudi Journal of Anaesthesia, 7(1), p.33.

Bell, L. (2015). American Association of Critical - Care Nurses, AACN Scope and Standards for Acute and Critical Care Nursing, Nurses books. Org, USA.

Botros, S., Mohamed, M., \& Ahamed, N. (2019). Assess nursing practice regarding safety measures on mechanically ventilated patients. Assiut Scientific Nursing Journal, 7(19), pp.48-57.

Critical Care Services Ontario. (2012). Standards for critical care nursing in ontario, Nurses books. org.

Dawson, D. (2005). Development of a new eye care guideline for critically ill patients. Intensive and Critical Care Nursing, 21(2), pp.119-122. 
Ebadi, A., Moayed, M.S., Mirsadeghi, A., \& Saeid, Y. (2021). Evaluating Intensive Care Nurses' Clinical Competence in Eye Care; a Cross-Sectional Descriptive Study. Health Education and Health Promotion, 9(3), pp.171-175.

Fashafsheh, I., Morsy, W., Ismaeel, M., \&Alkaias, A. (2013). Impact of a designed eye care protocol on nurses knowledge, practices, and eye health status of unconscious mechanically ventilated patients at North Palestine Hospitals. Journal of Education and Practice, 4 (28), pp.107-120. Retrieved from: https://www.iiste.org.

Fulbrook, P., Albarran, J., Baktoft, B., \& Sidebottom, B. (2012). A survey of European intensive care nurses' knowledge levels. International Journal of Nursing Studies, 49(2), pp.191200.

Grixti, A., Sadri, M., Edgar, J., \& Datta, A. (2012). Common ocular surface disorders in patients in intensive care units. The Ocular Surface, 10(1), pp.26-42.

Güler, E., Eşer, İ., \& Fashafsheh, I.(2017). Intensive care nurses' views and practices for eye care: An international comparison. Clinical Nursing Research, 26(4), pp.504-524.

Hu, J., Wang, Y., Chen, R., Zhang, X., Lin, J., Teng, J., \& Ding, X. (2017). Electrolyte and acid-base disturbances in critically ill patients: A retrospective and propensity-matched study. Int $J$ Clin Exp Med,10(1), pp.992-1003. DOI; ISSN:19405901/IJCEM0037834.
Huang, H., Chuang, Y., \& Chiang, K. (2009). Nurses' physical restraint knowledge, attitudes, and practices. Journal of Nursing Research, 17(4), pp.241-248.

Khalil, N., Abd Elhameed, S., AbdelKader, F., \& Atia, A. (2019). Critical care nurses' knowledge and practices concerning eye care of patients at two teaching university hospitals, Egypt. Nursing \& Healthcare International Journal, 3 (3).

Marshall, A., Elliott, R., Rolls, K., Schacht, S., \& Boyle, M. (2008). Eyecare in the critically ill: Clinical practice guideline. Australian Critical Care, 21(2), pp.97-109.

Mela, E., Drimtzias, E., Christofidou, M., Filos, K., Anastassiou, E., \& Gartaganis, S. (2010). Ocular surface bacterial colonization in sedated intensive care unit patients. Anesthesia and Intensive Care, 38(1), pp.190-193.

Milutinovic, D., Cvijanovic, D., Ciric, Z., Jovanovic, G., \& Andrijevic, A.(2017). Eyecare in mechanically ventilated critically ill adults-nursing practice analysis. Medical review, 70(1112), pp.377-383.

Mohamed, E., Abdl Elmoniem, A., El-Mowafi, H., \& Shebl, A. (2019). Effect of Training Program on Performance of Nurses Caring for Patient with Negative Pressure Wound Therapy. Journal of Nursing and Health Science, 8 (1) , pp. 31-35.

Rosenberg, J., \& Eisen, L. (2008). Eyecare in the intensive care unit: Narrative review and metaanalysis. Critical Care Medicine, 36(12), pp.3151-3155. 
Taha, N., \& Abd Elaziz, N. (2015). Effect of Nursing Intervention Guidelines on Nurses' Role, Patients' Needs, and Visual Problems Post Cataract Surgery. American Journal of Nursing Science, 4(5), pp. 261-269.

Vyas, S., Mahobia, A., \& Bawankure, S. (2018). Knowledge and practice patterns of Intensive Care Unit nurses towards eye care in Chhattisgarh state. Indian Journal of Ophthalmology, 66(9), p.1251.

Werli-Alvarenga, A., Ercole, F., Herdman, T., \& Chianca, $T$. (2012). Nursing interventions for adult intensive care patients with risk for corneal injury: A Systematic Review. International Journal of Nursing Knowledge, 24(1), pp.25-29.
Yim, W. (2009). Evidence-based eye care protocol for ICU patients with altered levels of consciousness. [online] Hdl.handle.net. Available at:

$<$ http://hdl.handle.net/10722/5696 6> [Accessed 3 February 2021].

Zanaty, M. M., Morsy. W. Y., Elshamy, K. F., \& Ali, S. A. (2016). Critical care nurses' knowledge and practices regarding sepsis bundle among critically ill patients at Mansoura Emergency Hospital. Master Thesis. Mansoura University, Faculty of nursing. 